\title{
Algorithm of power supply disturbance filtering for coal mine based on FRFT
}

\author{
Li-Kun Xing, Xiu-Ling Niu and Xin Li \\ An Hui University of Science \& Technology, Huai Nan, An Hui 232001, China \\ E-mail:1187455424@qq.com
}

\begin{abstract}
The environment of electricity for coal mine is very special, if there is something wrong with the power quality, it will cause great harm. The traditional Fourier transform algorithm makes the signal and noise overlap in the frequency domain and it has poor filtering performance when filtering in transient power quality disturbance signal. In order to solve this problem, the optimal filtering algorithm based on fractional Fourier transform (FRFT) is proposed. The algorithm adopts the optimal filtering principle under a minimum mean square error (MSE) criterion, using the obtained optimal order $p$ to filter, analyzing the filtered waveform and reconstructing the original signal. Through simulation results, it can be seen that FRFT has a better filtering effect and stronger self-adaptability in the treatment of coal mine power supply transient signal.
\end{abstract}

Keywords: FRFT; Transient Disturbance; Coal Mine Power Supply; Optimal Filtering.

\section{Introduction}

Power quality directly affects the safety of coal mine production, in the power supply system, more than $90 \%$ of the power quality problems are caused by voltage sag and raised. Voltage temporary drop is also called the voltage sag,, the effective value of the supply voltage has the phenomenon of reducing immediately and recovery in a certain period of time[1,2].

In this paper, the problem of voltage sag caused by short circuit fault in coal mine is analyzed and studied. When the signal is collected, because of various reasons, the signal can be interfered by noise. In order to obtain the pure original signal, the measured signal is processed by filtering. The fractional Fourier transform (FRFT) is a unified time-frequency transform, which can reflect the signal in time domain and frequency domain, and it has high time frequency aggregation. Compared with the traditional Fourier transform, its filtering effect is better[3]. 


\section{Fractional Fourier Transform (FRFT)}

\subsection{Definition}

FRFT is the counterclockwise rotation of the coordinate axis the signal in the plane of the frequency domain around the origin of the axis. If the ordinary Fourier transform is seen as an anticlockwise rotation of $\pi / 2$ from the time axis to the frequency axis, so the Fourier transform is a special case of FRFT [4]. Then FRFT can be regarded as an obtained transform that is an anticlockwise rotation of $\partial$ from the time axis to the $u$ axis [5]. Then the function $x(t)$ in the time domain is defined as the linear integral operator of the P order of FRFT:

$$
X_{p}(u)=\left\{F^{p}[x(t)]\right\}(u)=\int_{-\infty}^{+\infty} \tilde{K}_{P}(u, t) x(t) d t
$$

Among of which, $\tilde{k}_{p}(u, t)$ is called kernel function,

$$
\tilde{K}_{p}(u, t)=A_{\partial} \exp \left[j \pi\left(u^{2} \cot \alpha-2 u t \csc \alpha+t^{2} \cot \alpha\right)\right]
$$

The inverse transform of fractional Fourier transform can be regarded as the FRFT with order $-p$ That is:

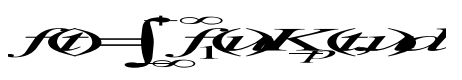

In practical applications, the discrete signal is usually treated, so matrix $F^{\partial}$ of DFRFT is defined as follows [6]:

$F^{\partial}=\left\{\begin{array}{l}\sum_{k=0}^{N-1} \exp (-j k \partial) v_{k} v_{k}{ }^{T}, N \text { is an odd number } \\ \sum_{k=0}^{N-2} \exp (-j k \partial) v_{k} v_{k}{ }^{T}+\exp (-j N \partial) v_{N-1} v_{N-1}{ }^{T}, N \text { is an even number }\end{array}\right.$

Among of which, $v_{k}$ is the characteristic vector of the approximate continuous Hermite-Gaussian function obtained from the $\mathrm{S}$ matrix.

\subsection{FRFT domain optimal filtering algorithm}

In the process of processing noise signals, if the noise and the signal does not exist time frequency coupling, the coupling of noise and signal can be eliminate by means of coordinate rotation. It is also called multiplicative filter as shown in Figure 1. But when a distortion system causes distortion of the signal or the time frequency coupling cannot be eliminated by means of coordinate rotation, a 
more general filtering method can be used, that is, the optimal filtering in the criterion of minimum mean square error [7].

For the power quality disturbance signal containing noise, the projection of the original signal and the noise in the time axis and the frequency axis are likely to overlap. In the $U$ domain of FRFT, the original signal has different degrees of aggregation because of $\mathrm{P}$, but the noise signal has no aggregation whenever. Therefore, there will be an optimal $\mathrm{P}$ order in the $\mathrm{U}$ domain to make the most useful aggregation of signal. In this way, the coupling effect of noise on the useful signal can be reduced or eliminated [8].

Assuming the electricity signal with noise is:

$$
x(t)=s(t)+n(t)
$$

Among of which, $s(t)$ stands for original signal and $n(t)$ stands for interference signal. Due to the superposition of FRFT, FRFT can be simultaneously conducted for above formula:

$$
X_{p}(u)=S_{p}(u)+N_{p}(u)
$$

Here, respectively stands for discrete FRFT of $x(u), s(u), s(u)$.p stands for the order of FRFT. The least mean square error (MSE) is used as a criterion to measure the effectiveness of the filter in order to better eliminate the effect of noise on the useful electricity signal. $s(n), s 1(n)$ respectively stands for the original signal and signal after filtered processing. The smaller the value of MSE is, the better the filtering effect is, and the formula of MSE is as follows:

$$
\sigma_{e, 0}^{2}=E \sum_{N=0}^{N-1}[s(n)-s 1(n)]^{2}
$$

According to the simulation results of $\mathrm{P}$ and MSE, we can know that there is an order $\mathrm{P}$ in the FRFT transform interval to minimize the MSE. Therefore, according to the MSE criterion, we can obtain the optimal transformation order p. In this order, the measured signal is processed by filtering, and then the $P$ Order Fractional Fourier transform to the processed waveform can be done, so we can obtain the filtered signal [9].

So the general process of optimal filtering under MSE criterion is:

(1) to estimate the parameters of the original signal, to obtain the optimal order based on the principle of least mean square error

(2) to do P0 order FRFT of the signal, and according to the measured signal to set the appropriate window function $M(u)$ to filter;

(3) to do the inverse FRFT of the filtered signal, to analysis and evaluation 
of the disturbance signal after the inverter.

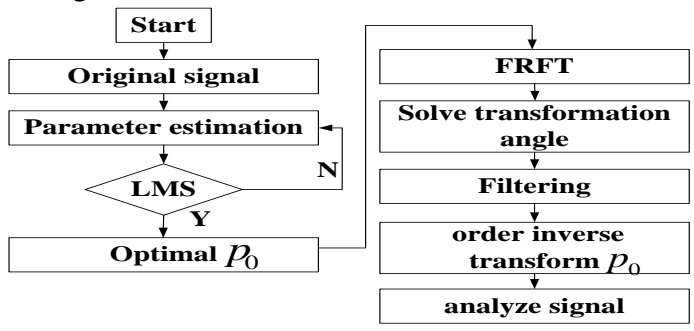

Fig. 1 Filtering flow chart based on FRFT

The computation complexity of FRFT denoising is compared with that of the traditional Fourier transform in frequency domain denoising. Comparing to the traditional Fourier transform, FRFT has a free parameter, which is a linear transformation. Because there is no cross interference, FRFT is more universal in the decoupling filter [10].

\section{Simulation Analyses}

\subsection{Coal mine power supply transient disturbance simulation}

Using MATLAB/Simulink software to simulate the phenomenon of short circuit voltage drop of power supply in coal mine.

To do fractional Fourier transform for voltage sag signal generated by single phase short circuit fault. In the $\mathrm{U}$ domain, the 0.01 step is used to find the optimal order, and then the measured signal is treated as the $p_{0}$ order fractional Fourier transform. Here we assume that the single phase to ground fault signal contains noise, and the noise in the system is simulated by adding white noise (assuming a signal to noise ratio of 10). The MSE waveforms for FRFT are as follows:

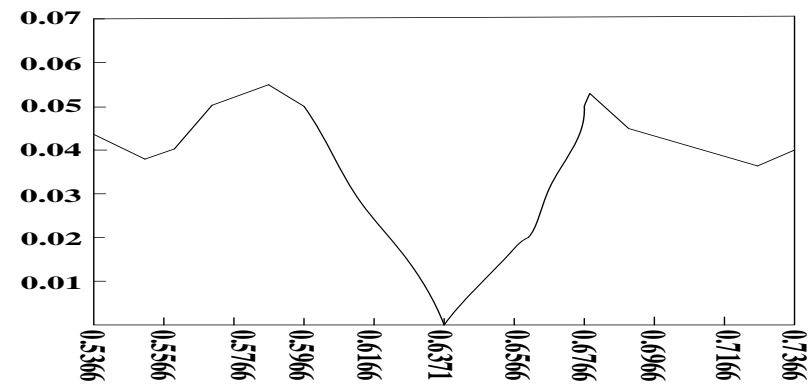

Fig. 2 changes of $\mathrm{P}$ with MSE graphic 
From the above graphic, when $\mathrm{p}$ is equal to 0.6371 , the amplitude of the MSE is the smallest, and the filter of FRFT is the best. Related waveforms are as follows:
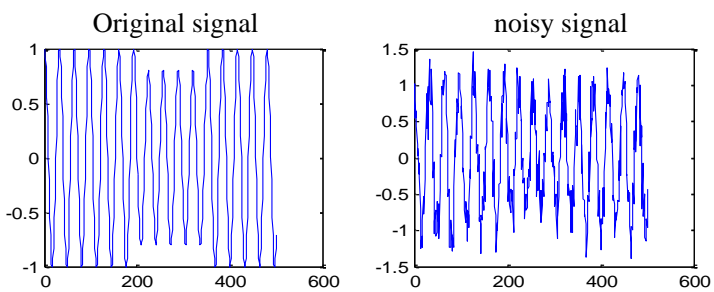

Fig. 3 Waveform before and after adding noise

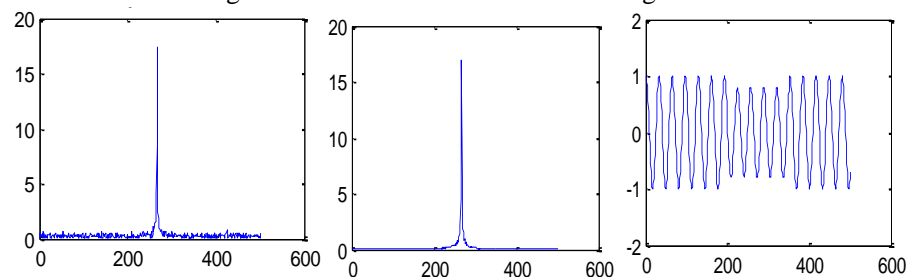

$\begin{array}{lll}\text { (a) FRFT at optimal order } & \text { (b) Filtered FRFT } & \text { (c) Filtered waveform }\end{array}$

Fig. 4 Optimal FRFT and filter graph

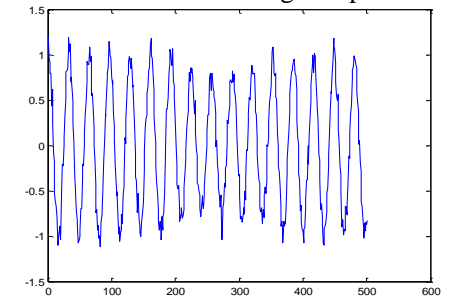

Fig. 5 The waveform after FFT filtering

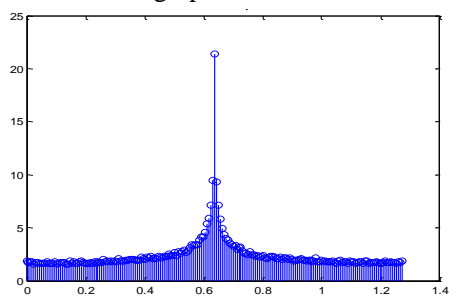

Fig. 6 spectral peak diagram of FRFT

\subsection{Simulation results analysis}

Fig. 2 is the MSE changes in graphics with the order of the $\mathrm{P}$, said that at a point of $\mathrm{P}$ value, the MSE amplitude is the smallest; Fig6 is the spectral peak of the signal at different orders of $\mathrm{P}$. Combined with two figures to analyze, when $\mathrm{p}$ is about at $0.6371, \mathrm{MSE}$ is the smallest, spectral peak value is the largest and the focus is also the best. Combining the smoothness of the waveform after FFT filtering in Fig 4 with that after FRFT filtering in Fig 5, it can be clearly seen that the filtering effect of FRFT is better.

Fig. 7 is the comparison of the filtering effect of the two algorithms. The effect of filtering algorithm can be measured by the number of signal to noise ratio, and signal to noise ratio is the ratio of the useful signal to the noise signal. 
The input signal to noise ratio( $\left.S N R_{\text {in }}\right)$ before filtering and the output signal to noise ratio $\left(s n r_{\text {out }}\right)$ after filtering Under the premise of the same input signal to noise ratio, the greater the output signal to noise ratio is, the better the filtering effect is, so the FRFT filtering effect is better.

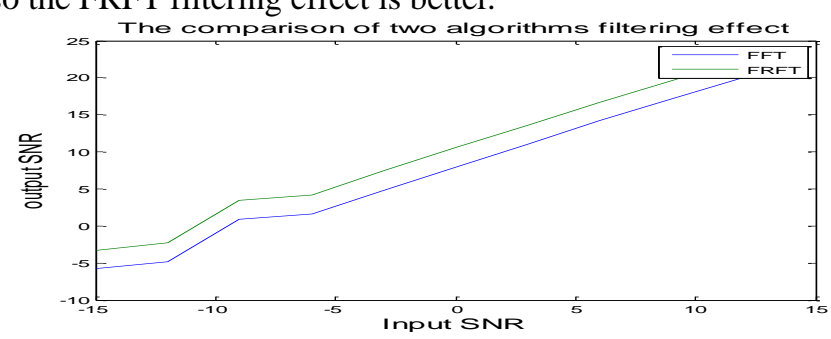

Fig. 7 comparison of filtering effects of FRFT and FFT algorithm

Tab. 1 The effect of fractional order $\mathrm{P}$ on the $s n r_{\text {out }}$

\begin{tabular}{|c|c|c|c|c|c|}
\hline $\mathrm{p}$ & 0.1 & 0.2 & 0.3 & 0.4 & 0.5 \\
\hline \multirow{2}{*}{ Snr } & 17.7912 & 18.3465 & 18.0561 & 17.3543 & 17.7306 \\
\hline $\mathrm{p}$ & & & & & \\
\hline \multirow{2}{*}{ sut } & 0.6371 & 0.7 & 0.8 & 0.9 & 1.0 \\
\hline out & 19.6368 & 17.3100 & 16.8752 & 18.6107 & 17.7757 \\
\hline
\end{tabular}

In Table 1 when the $S N R_{\text {in }}$ is invariant, with the increase of order $\mathrm{p}$, the $s n r_{\text {out }}$ firstly increase then decrease. So there is an optimal order $\mathrm{p}$ can make the $s n r_{\text {out }}$ maximum and make the corresponding MSE minimum, and the filtering effect is best. For the transient power quality disturbance signal, after FRFT treatment, the aggregation of different signal is different. In the optimal u field corresponding to the optimal order, the noise signal is no aggregation, noise and useful signals is at the minimum coupling, and the decoupling filtering effect is the best.After the similar processing of other non-stable signal, then we can effectively control and manage the power quality problems of all kinds of power systems, and then improve and guarantee the power supply quality of the coal mine.

\section{Conclusion}

In this paper, a power supply disturbance filter algorithm based on FRFT is proposed. Under the minimum mean square error criterion we can obtain the optimal transform order, using the aggregation of fractional Fourier transform to separate the noise and useful signal and to achieve the purpose of decoupling. The simulation results show that FRFT has better filtering effect of the transient disturbance signal in coal mine power supply than the traditional Fourier 
transform, and FRFT has accurate description and evaluation of it, which provides a powerful guarantee for the safe production of coal mine.

\section{References}

1. Hao.L.B.In the production of coal mine power quality analysis[J].Shandong Coal Science and Technology,2008,04:109-110..(In Chinese).

2. li Y Y.Algorithm of an optimal filter based on fractional domain[J]. ELECTRONIC TEST, 2011, 25(8):23-26.(In Chinese).

3. Tang Z M, Yang G Q, Xu H L.A Method for Speech Enhancement Based on fractional domain optimal filtering $[\mathrm{J}]$. Information \& communications, 2011(01):12-14..(In Chinese).

4. Tao R,Deng B,Wang Y. Fractional Fourier Transform and Its Applications[M]. Bei Jing: Tsinghua University Press 2009:12-132.(In Chinese).

5. V.Namias. The fraction order Fourier transform and its application to quantum mechanics [J]. J of Appl Math, 1980, 25:241-265.

6. H. M. Ozaktas and B. Barshan. D Mendlovic and LOnural. Convolution, Filtering, and Multiplexing in fractional Fourier Domains and their relation to Chirp and Wavelet Transforms [J]. J. Opt. Soc . Amer. A. , 1994, 11(2) : 547-559.

7. Xu F W, Liu Y M, Yang H G. Voltage sags identification based on generalized S-transform[J]. Power System Protection and Control, 2013, 41(17):18-24..(In Chinese).

8. V. A shok Narayanan, K. M. M. Prabhu. The Fractional Fourier transform :Theory, Implementation and Eorr Analysis[J]. Microprocessors and Microsystems, 2003, 27, 5111-521.

9. Wang Z F. Electrical Power Quality Disturbance Analysis Based On S Transform And Wavelet Denoising[D]. Harbin: Yanshan University, 2015:9-18..(In Chinese).

10. Zhang X S. Coal Mine power quality analysis and control[J]. Industry and Mine Automation, 2009, 35(09): 68-70..(In Chinese). 